\title{
Adhesive hysteresis and rolling friction in rough "non-adhesive" contacts
}

\author{
Valentin L. Popov \\ Technische Universität Berlin, Str. des 17. Juni 135, 10623 Berlin, Germany \\ v.popov@tu-berlin.de
}

\begin{abstract}
In 1975, Fuller and Tabor have shown that roughness can destroy macroscopic adhesion. This means that in spite of the presence of adhesion at the microscopic scale, the macrosopic force of adhesion vanishes. The mechanism of vanishing macroscopic adhesion is very simple: during approach of elastic bodies, asperities are elastically deformed so strongly that after unloading they destroy the microscopic adhesive junctions. However, both in the moment of formation of microscopic adhesive junctions in the loading phase and their destruction during unloading, mechanical energy disappears. This means that the microscopic adhesion makes the contact dissipative even if there is no macroscopic force of adhesion. In particular, the force-distance dependency during indentation and pull-off do not coincide with each other showing some "adhesive hysteresis". When a ball rolls on such rough surface, there will be a final energy dissipation due to formation of a new contact at the frontline of the contact and its destruction at the rear part. Thus, microscopic adhesion will lead to appearance of rolling friction in an apparently non-adhesive contact. In the present paper, we calculate the approach and pull-off dependencies of force on distance, the dissipated energy in one loading-unloading cycle and estimate the force of rolling friction due to microscopic adhesion.
\end{abstract}

Keywords: Adhesion, hysteresis, energy dissipation, JKR theory, rolling friction

\section{Introduction}

In 1949, Bowden and Tabor proposed a concept, explaining the origin of sliding friction through formation and shearing of adhesive junctions [1]. Initially, this concept was criticized, because formation of adhesive junctions apparently had to lead to finite adhesive force, which normally is not observed in contacts of stiff bodies (as e.g. metals or stiff rubber). The paradox was resolved by Fuller and Tabor in 1975 [2]. They have shown that the adhesive force can vanish completely if surfaces are "rough enough". The mechanism of vanishing macroscopic adhesion is very simple: during approach of elastic bodies, asperities are so strongly elastically deformed that after unloading they stored elastic energy is enough for destroying the microscopic adhesive junctions. Fuller and Tabor considered a statistical distribution of independent "asperities" (with characteristic "roughness" l) having constant stiffness and adhesive properties characterized by a characteristic length $\Delta h_{\text {adh }}$, by which each single asperity can expand before it separates from the surface. This model belongs to the same category as the famous Greenwood and Williamson model of rough surfaces [3], with the only differences that it was extended by adhesion. Fuller and Tabor have shown that the adhesive properties are determined by the parameter $\Delta h_{\text {adh }} / l$. If $\Delta h_{\text {adh }} / l>1$, the adhesion force is proportional to the applied normal force (thus presenting a "pressure sensitive adhesive"), while if $\Delta h_{\text {adh }} / l<1$, the adhesive force vanishes completely. The derivation of these properties is reproduced in a compact way in [4] (pp. 113-114) and will be followed further in the present paper.

Just as the Greenwood and Williamson model, the model by Fuller and Tabor does not consider elastic interaction of asperities. $\mathrm{Li}$ and Popov studied in [5] this interaction numerically using the Boundary Element Method adapted for adhesive contacts [6],[7] in the approximation of short range adhesive interaction (Johnson, Kendall and Roberts, JKR [8],[9]). Li and Popov have shown that the Fuller and Tabor model overestimates the adhesive force. However, the qualitative behavior of the contacts remains the same: The adhesive force vanishes if the roughness exceeds substantially 
the adhesive length $\Delta h_{\text {adh }}$. Thus, the model of Fuller and Tabor is not exact but it can be used for qualitative analysis. In the present paper, we use for simplicity this model.

Physically similar situation, only in the framework of a continuum mechanics model, was considered by Johnson in 1995 [10]. Johnson studied an adhesive contact between a wavy surface with amplitude $l_{0}$ and wavelength $\lambda$ :

$$
f(x)=l_{0}[1-\cos (2 \pi x / \lambda)]
$$

and an elastic half space with effective elastic modulus $E^{*}=E /\left(1-v^{2}\right)$, where $E$ is elastic modulus and $v$ is Poisson's ratio. He has shown that the behavior of the contact is determined by the dimensionless parameter $\alpha$ defined as

$$
\alpha=\left(\frac{2 \lambda \gamma}{\pi^{2} l_{0}^{2} E^{*}}\right)^{1 / 2},
$$

where $\gamma$ is the work of adhesion per unit area. In the following, we will refer to $\alpha$ as the "Johnson parameter". At the values of Johnson parameter $\alpha>0.57$ the system jumps into complete contact immediately at first touch. Thus, for $\alpha \gg 0.57$ the surfaces can be considered as smooth. In the opposite case of $\alpha \ll 0.57$, the contact occurs only on the summits, and we have a "practically nonadhesive" contact. In reality, Johnson parameter is closely related to the above Fuller and Tabor parameter. One can show that

$$
\alpha \approx\left(\frac{\Delta h_{\text {adh }}}{l_{0}}\right)^{3 / 4} .
$$

In terms of Johnson parameter, the present paper focuses mainly on the range of small values of the Johnson-parameter (and thus practically non-adhesive) system.

\section{Problem statement and model description}

The considered system is schematically shown in Fig. 1. It is composed of springs (total number $N_{0}$ ) with the stiffness $k$, which can adhere if brought into contact. Their adhesive properties are characterized by the length $\Delta h_{\text {adh }}$, by which a spring can expand before it separates from the surface. Let the height distribution of the springs be

$$
\Phi(z)=\frac{1}{l} e^{-\frac{z}{l}}
$$

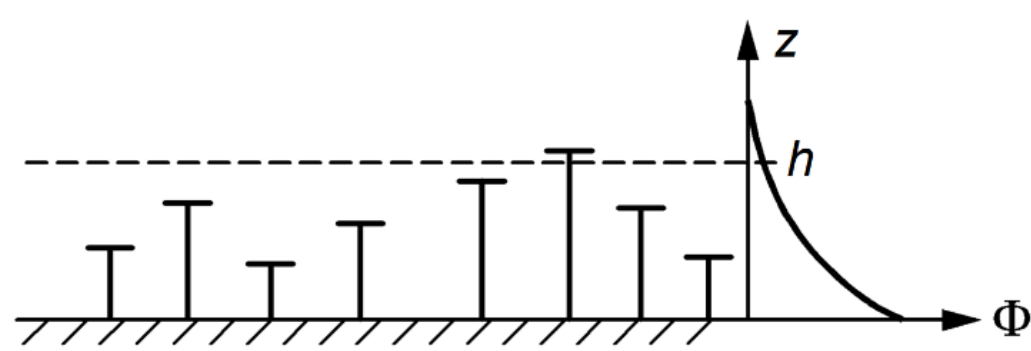

Fig. 1 Spring model of a stochastic elastic surface.

A rigid plane (shown in Fig. 1 with dashed line) initially approaches the system from infinity, is pressed up to the height $\tilde{h}$ and then pulled away to a distance of $h$. During the approach phase, 
when the plane is at the height $h$, only those springs with a height of $z>h$ come in contact with the plane. The normal force, $F_{N}$, can be calculated as

$$
F_{N}=\int_{h}^{\infty} \frac{N_{0}}{l} e^{-\frac{z}{l}} k(z-h) d z=N_{0} k l e^{\frac{-h}{l}} .
$$

In particular, the maximum force (at the height $\tilde{h}$ ) is equal to

$$
F_{N, \max }=\int_{h}^{\infty} \frac{N_{0}}{l} e^{-\frac{z}{l}} k(z-\tilde{h}) d z=N_{0} k l e^{\frac{-\tilde{h}}{l}} .
$$

If during the pull-off the position of the rigid plane is changed from $\tilde{h}$ to $h$, then all of the springs with heights in the non-deformed state larger than $h-\Delta h_{\text {adh }}$ and larger than $\tilde{h}$ will remain in contact. The force acting on the plane is equal to

$$
\begin{array}{cl}
F_{N}=\int_{\tilde{h}}^{\infty} \frac{N_{0}}{l} e^{-\frac{z}{l}} k(z-h) d z=N_{0} k l e^{\frac{-\tilde{h}}{l}}\left(1+\frac{\tilde{h}}{l}-\frac{h}{l}\right), \quad \text { for } \quad h<\tilde{h}+\Delta h_{\text {adh }} \\
F_{N}=\int_{d-\Delta h_{\text {adh }}}^{\infty} \frac{N_{0}}{l} e^{-\frac{z}{l}} k(z-h) d z=N_{0} k l e^{\frac{\Delta h_{\text {adh }}-h}{l}}\left(1-\frac{\Delta h_{\text {adh }}}{l}\right), & \text { for } \quad h>\tilde{h}+\Delta h_{\text {adh }}
\end{array}
$$

Eq. (5),(7) and (8) describe completely both the phases of approach and pull-off.

\section{Force-displacement relations}

(I) For $\Delta h_{\text {adh }} / l<1$, the force acting on the plane is always positive, meaning that there is no macroscopic adhesion.

(II) For $\Delta h_{\text {adh }} / l>1$, the absolute value of the negative force reaches its maximum value for $h=\tilde{h}+\Delta h_{\text {adh }}$. This value is the adhesive force:

$$
\left|F_{A}\right|=N_{0} k l e^{-\frac{\tilde{h}}{l}}\left(\frac{\Delta h_{\mathrm{adh}}}{l}-1\right) .
$$

In this model, the ratio of adhesive force to compressive force,

$$
\frac{\left|F_{A}\right|}{F_{N}}=\frac{\Delta h_{\mathrm{adh}}}{l}-1,
$$

is not dependent on the compressive force and is called the adhesion coefficient. For $l=\Delta h_{\mathrm{adh}}$, the adhesive force becomes zero.

The dependencies of the normal force on the "indentation depth" (which we define just as the distance $h$ with negative sign: $d=-h$ ), are shown in Fig. 2. Curve (1) corresponds to the phase of approach. In this model, it does not depend on the strength of adhesion. After reversal of the movement from approach to pull-off, the dependency is first linear, described by Eq. (7) and shown in Fig. 2 with dashed line up to the point $h=\tilde{h}+\Delta h_{\text {adh }}$. After that, it is defined by Eq. (8) and shown in Fig. 2 with color curves corresponding to four values of the Fuller-Tabor parameter $\Delta h_{\text {adh }} / l$. The red curve (3) corresponds to the critical value $\Delta h_{\text {adh }} / l=1$ when the macroscopic adhesion vanishes for the first time. Interestingly, in this critical state, the normal force vanishes already at a final 
distance and remains zero after that - a behavior that we would await if there were some plastic deformation. In reality, we have to do with an ideally elastic system.

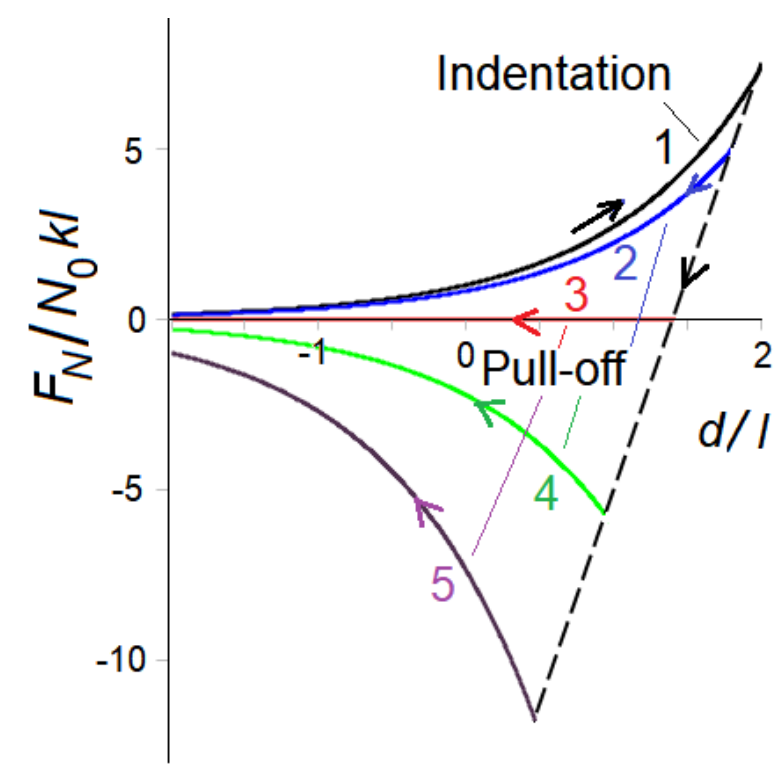

Fig. 2 Dependencies of the normal force on the indentation depth. Curve (1) corresponds to the approach (independently on the strength of adhesion). The blue curve (2) corresponds to small enough adhesion (or large enough roughness) with no macroscopic force of adhesion. The red curve (3) corresponds to the critical state when the force of adhesion vanishes. The green curve (4) and the curve (5) correspond to the final force of adhesion. The values of the Fuller-Tabor parameter, $\Delta h_{\text {adh }} / l$, are: (1) 0, (2) 0.5, (3) 1.0, (4) 1.5, (5) 2.0 .

Understandingly, the curves shown in Fig. 2 are idealizations valid only if the statistics of asperities is big enough. Full 3D numerical simulations of a similar system of randomly distributed cylindrical "asperities" shown in Fig. 3 demonstrate that the above simple statistical model describes qualitative well the overall behavior of the system. In particular, one can see the linear part of the dependency after reversal of the direction of motion and the adhesion hysteresis. However, the dependency after achievement of the force of adhesion differs in details.

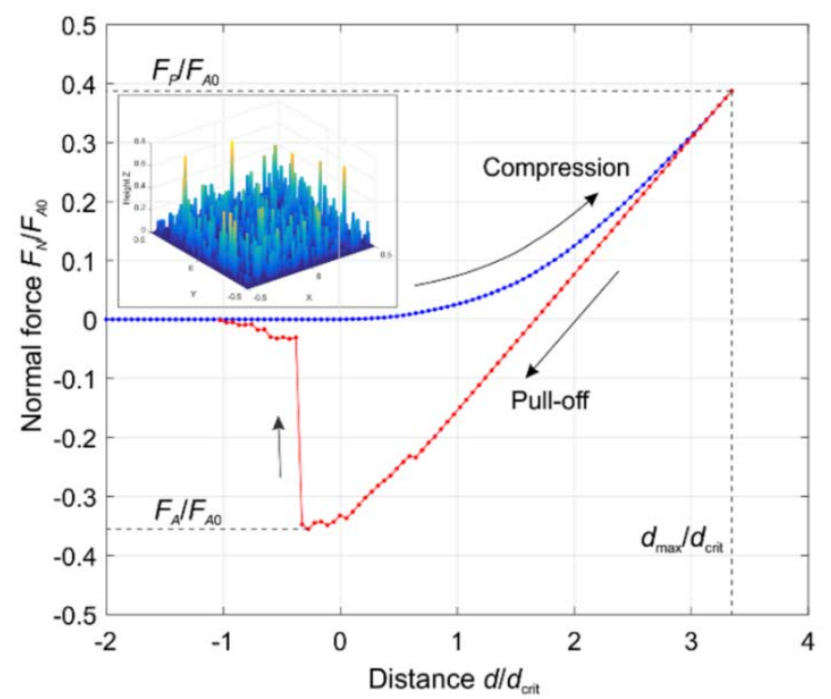

Fig. 3 Dependence of the normal force on indentation depth for a rough "brush"-shaped indenter. Results have been obtained in [5] with full 3D Boundary Element simulations. 


\section{Energy dissipation}

Let us now consider one full cycle of contact starting in the infinity, approaching the rough surface until the height $\tilde{h}$ and going back to the infinity. The total work performed by the normal force during the cycle will be given by a sum of integrals of three stages of approach and pull off

$$
W=\underbrace{\int_{\infty}^{\tilde{h}} F_{N}(h) \mathrm{d} h}_{E q . \text { (5) }}+\underbrace{\int_{\tilde{h}}^{\tilde{h}+\Delta h_{\mathrm{adh}}} F_{N}(h) \mathrm{d} h}_{E q .(7)}+\underbrace{\int_{\tilde{h}+\Delta h_{\mathrm{adh}}}^{\infty} F_{N}(h) \mathrm{d} h}_{E q .(8)},
$$

while the normal force is defined by one of Eqs. (5), (7), and (8). Performing integration gives

$$
W=-\frac{1}{2} N_{0} k \cdot e^{-\tilde{h} / l} \cdot\left(\Delta h_{\mathrm{adh}}\right)^{2} .
$$

The work is negative corresponding to energy dissipation. This equation can be derived also without integration. If we consider one single spring which is tensed up to elongation $\Delta h_{\text {adh }}$, then the elastic energy stored in it in the moment of detachment is equal to $\frac{1}{2} k \Delta h_{\text {adh }}^{2}$. Due to detachment, all this energy gets lost. The number of springs coming into contact can be easily calculated as $N_{0} e^{-\tilde{h} / l}$. Multiplying energy lost per spring with the number of springs gives (12). This mechanism of dissipation reminds the "relaxation damping" described in [11].

With account of (6), the amount of dissipated energy can be rewritten in the form

$$
|W|=\frac{F_{N, \max } \cdot \Delta h_{\mathrm{adh}}^{2}}{2 l} .
$$

Note that the dissipated energy is a linear function of the normal force. This means that it will be correct not only for the plane geometry considered above but also for a contact of an arbitrary shape with a macroscopically homogeneous rough surface. Thus, the validity of Eq. (13) is wider than the assumptions under which it was derived.

Note further that the equation (13) does not make difference between "adhesive" and "nonadhesive" contacts: It is valid for both. For us it is important to stress that the dissipated energy is non-zero even in the case of vanishing macroscopic adhesion.

\section{Rolling Friction}

We use the above-derived equation for the dissipated energy for estimating the rolling friction due to adhesion. This is easily possible because the rolling contact is basically the normal contact (while at the front boundary the new contact is created and the rare boundary destroyed). The complete cycle of indentation and detachment occurs when the rolling ball is moved by the contact diameter, $2 a$. The force of friction can be calculated as

$$
F_{\mathrm{Fr}} \approx \frac{|W|}{2 a}=\frac{F_{N, \max } \cdot \Delta h_{\mathrm{adh}}^{2}}{4 a l} .
$$

For the coefficient of rolling friction, $\mu$, we get

$$
\mu=\frac{F_{\mathrm{Fr}}}{F_{N, \max }}=\frac{\Delta h_{\mathrm{adh}}^{2}}{4 a l} .
$$


Let us finally estimate the coefficient of friction in terms of the Johnson parameter. For this sake, assume that we have a surface characterized not by a single wavelength as (1) but by a spectrum with the average wavelength $\lambda$ and standard variation of the same order of magnitude. We then have to do with a stochastic surface, which, however, still can be characterized by its central wavelength $\lambda$. Taking into account the estimation (3), we can rewrite the coefficient of friction in the form

$$
\mu \approx \frac{l}{4 a} \alpha^{4 / 3} .
$$

The rolling coefficient of friction due to adhesion is very small. Indeed, in almost any real situation $l / a \ll 1$. In a non-adhesive contact the Johnson parameter is also small $\alpha \ll 1$. A specific assessment can of course be done only for a specific system.

\section{Conclusions}

We studied energy dissipation in rough adhesive contact with over-critical roughness, so that the macroscopic force of adhesion vanishes. While macroscopically such contacts can appear as "non-adhesive", the adhesion on the microscale continues to play an important role and, in particular, leads to energy dissipation due to formation and destruction of microscopic adhesive junctions. For our analysis, we used the model suggested by Fuller and Tabor. While the results obtained are formally valid for any adhesion intensity, the applicability of the Fuller and Tabor model, in our opinion, is restricted to the non-adhesive case (or to the limit of small values of Johnson parameter). That is why we would not like to claim that the obtained results is universal, but it surely is a good approximation for the cases when the contact is not a compact area but have the shape of a cloud of contact spots. In this approximation, the result for energy dissipation is amazingly simple: It is proportional to the applied force, to the square of the characteristic detachment distance and inversely proportional to the roughness.

This result allows deriving the coefficient of rolling friction as rolling is basically a normal contact. The coefficient of friction is given by Eq. (15): it is ratio of the square of detachment length and the product of the contact radius with roughness.

\section{References}

[1] Bowden F P, Tabor D. The Friction and Lubrication of Solids. Clarendon Press, 2001

[2] Fuller K N G, Tabor D. Proc. R. Soc. A Math. Phys. Eng. Sci., 345 (1642), 327-342 (1975). doi:10.1098/rspa.1975.0138

[3] Greenwood J A, Williamson J B P. Proc. R. Soc. A Math. Phys. Eng. Sci. 295 (1442), 300319 (1966). doi: 10.1098/rspa.1966.0242

[4] Popov V L. Contact Mechanics and Friction. Physical Principles and Applications. 2nd Edition. Berlin: Springer, 2017

[5] Li Q, Popov V L. Adhesive contact of rough brushes, Beilstein journal of nanotechnology, 9 (1), 2405-2412 (2018). doi:10.3762/bjnano.9.225

[6] Popov V L, Pohrt R, Li Q. Friction, 5 (3), 308-325 (2017). doi:10.1007/s40544-017-0177-3

[7] Popova E, Popov V L. Note on the history of contact mechanics and friction: Interplay of electrostatics, theory of gravitation and elasticity from Coulomb to Johnson-Kendall-Roberts theory of adhesion, Physical Mesomechanics, $21 \quad$ (1), 1-5 (2018). https://doi.org/10.1134/S1029959918010010 
[8] Johnson K L, Kendall K, Roberts A D. Surface Energy and the Contact of Elastic Solids. Proceedings of the Royal Society of London, Series A, 324, 301-313 (1971).

[9] Kendall K. The adhesion and surface energy of elastic solids, J. Phys. D. Appl. Phys. 4 (8), 1186 (1971). doi:10.1088/0022-3727/4/8/320

[10] Johnson K L. The adhesion of two elastic bodies with slightly wavy surfaces, Int. J. Solids Struct., 32, 423-430 (1995).

[11] Popov M, Popov V.L., Pohrt R, Relaxation damping in oscillating contacts, Scientific reports 5, 16189 (2015). https://doi.org/10.1038/srep16189 